\title{
A PCR-based microwell-plate hybrid capture assay for high-risk human papillomavirus
}

\author{
Yumei Wang • Yan Liu • Yaping Ding • \\ Nan Sun $\cdot$ Yafang Gong $\cdot$ Shangxian Gao
}

Received: 28 February 2014/ Accepted: 17 July 2014/Published online: 5 August 2014

(C) The Author(s) 2014. This article is published with open access at Springerlink.com

\begin{abstract}
Human papillomavirus (HPV) is associated with cervical cancer. In this study, we developed a highthroughput microwell-plate hybrid capture (MPHC) method for epidemiological studies of high-risk HPV (HRHPV). The results with 1238 cervical specimens from female outpatients showed a concordance rate of $94.3 \%$ between the MPHC and Hybrid Capture II assay. The MPHC assay showed an average HRHPV rate of $29.3 \%$ for high-risk populations in populous cities of China. The established MPHC assay could sensitively and specifically detect 13 types of HRHPV and is suitable for large-scale screening, especially in areas where real-time PCR or fluorescence equipment is unavailable.
\end{abstract}

It is well established that persistent infection with high-risk human papillomaviruses (HPV) plays the leading etiological role in the development of cervical and anal cancer and their immediate precursors [1-3]. Based on sequence

Electronic supplementary material The online version of this article (doi:10.1007/s00705-014-2186-0) contains supplementary material, which is available to authorized users.

Y. Wang $\cdot$ Y. Liu $\cdot$ N. Sun $\cdot$ S. Gao $(\bowtie)$

Department of In Vitro Diagnostic Reagents, Key Laboratory of the Ministry of Health for Research on Quality and

Standardization of Biotech Products, National Institutes for Food and Drug Control, No. 2 Tiantanxili, Beijing 100050, China

e-mail: gaoshangxian@nifdc.org.cn

\section{Y. Ding}

Shenzhen Kangmei Biotechnology Co., Ltd, Shenzhen 518057, China

Y. Gong

Third Xiangya Hospital of Central South University,

Changsha 410013, China differences within their L1 gene, HPVs are divided into more than 100 different genotypes, in which approximately 40 types of HPV transmitted through the genital tract have been classified further as "low-risk" and "high-risk" based on their association with malignant lesions [4]. The worldwide prevalence of high-risk human papillomavirus (HRHPV) infection in women without cervical abnormalities ranges from 0 to $48.4 \%$, and in developing countries, such as the countries of Asia and sub-Saharan Africa, the prevalence is higher than that in developed countries [5-7]. China is currently estimated to have 130,000 new cervical cancer cases each year, accounting for $28 \%$ of the world's total [8]. Therefore, a high-throughput assay is urgently needed for assessing the level of the HRHPV epidemic in current populations for the diagnosis and treatment of cervical cancer [9-11].

Several HPV detection methods, including PCR-based methods and the Hybrid Capture II-based system, have been established to assess the presence of 13 high-risk HRHPVs [12-14]. In these methods, several consensus or degenerate primer pairs have been designed to amplify the relatively conserved region of the L1 gene in the HPV genome, including GP5/GP6 and PGMY09/PGMY11 [15, 16]. Although these consensus or degenerate primers could amplify a wide spectrum of HPV genotypes, the sensitivity and specificity of different primer sets to detect HPV DNA need to be evaluated in a clinical study, and the overall prevalence of HPV needs to be properly estimated by a variety of detection methods [16, 17]. Moreover, neither cytology-based screening nor molecular tests for HRHPV are widely available in developing countries, which lack the necessary human, financial, and material resources, including instruments such as real-time PCR equipment and fluorescence detectors. Therefore, it is essential to develop a low-cost, high-throughput screening method to 
test large numbers of patients in developing countries [18]. In this paper, we describe a sensitive, low-cost, highthroughput and easily operated microwell-plate hybrid capture (MPHC) assay that detects 13 HRHPV types using a novel set of primers that specifically bind to the L1 region.

For the MPHC system, the YR8/YR10 primer set was used, which contains eight forward primers and nine reverse primers. Both primer pools were conjugated with biotin at their $5^{\prime}$ ends. Two segments of the amplified sequence, one each from the sense and antisense strands, were selected as the capture probes. Each probe had an oligo-T linker modified with an amino group at its $5^{\prime}$ end. The sequences of all the primers and the probes and their GenBank accession numbers are shown in Table 1. Twenty-six HPV probe mixtures were immobilized to activated microwells. Compared with the PGMY11/ PGMY09 consensus primer set, which produces a product of $450 \mathrm{bp}$ [16], the YR8/YR10 primer set produces a shorter product of $185 \mathrm{bp}$, which reduces the spatial obstacles to hybridization, thus improving the overall efficiency of hybridization.

For the establishment and validation of the MPHC assay, the HPV DNA was amplified with the YR8/YR10 primer set and the biotin-conjugated products were added to the probecoated microwells. After alkaline denaturation, neutralization, hybridization and washing, horseradish-peroxidaseconjugated streptavidin (Dako) was used to bind target PCR products, and $3^{\prime}, 3^{\prime}, 5,5^{\prime}$-tetramethylbenzidine (SigmaAldrich) was then used to develop the color reaction. The optical density at $450 \mathrm{~nm}\left(\mathrm{OD}_{450}\right)$ of each well was measured using an enzyme-linked immunosorbent assay plate reader (SpectraMax M5).

The negative and positive cutoff values for the MPHC method were first estimated from 400 samples that were identified as negative by calculating the mean negative $\mathrm{OD}_{450}$ values plus two or three standard deviations to be 0.56 and 0.70 , respectively (Fig. 1A). The cutoff values were then validated with another 100 specimens identified with the HCII assay (50\% negative and $50 \%$ positive). It was found that the $\mathrm{OD}_{450}$ values of more than $95 \%$ of the negative samples were lower than 0.56 , and more than $95 \%$ of the positive samples had values higher than 0.70 (Fig. 1B). Therefore, the equivocal zone was between 0.56 and 0.70 . Ambiguous specimens were tested again in duplicate and were defined as positive when one result was positive or two results were equivocal in these duplicate reactions. Otherwise, when the value of a specimen that is suspected to be infectious is in the range of $0.70-1.0$, we suggest that a second specimen be analyzed and/or an alternative testing method be used, especially for liquid-preserved cytology specimens.
In order to determine the sensitivity and specificity of MPHC, 43 types of HPV (types 16, 18, 31, 33, 35, 39, 45, 51, $52,56,58,59,68,6,11,26,34,40,42,43,44,53,54,55,57$, $62,64,66,67,69,70,71,72,73,79,80,81,82,83,84,87,90$, and 91) plasmids were constructed. The sequence information for the HPVs was obtained from the GenBank database. To determine the detection limits of the MPHC for HPV DNA, a serial 10-fold dilution of each HRHPV plasmid and human genomic DNA from HPV18-transformed cells (PZHPV-7, ATCC CRL-2221D) was tested and analyzed using the MPHC assay to determine the positive cutoff concentration. To further determine the specificity of the assay, the assay was tested against DNA from viral strains, bacterial cultures, and clinical samples, including cytomegalovirus, herpes simplex virus 2, Chlamydia trachomatis, Ureaplasma urealyticum, Neisseria gonorrhoeae, Mycoplasma humenis, M. genitalium, E. coli, Bacillus pyocyaneus, Staphylococcus aureus, Candida albicans, and Treponema pallidum. The results showed that all $13 \mathrm{HRHPV}$ plasmids were detected as positive at concentrations of $10^{3}$ copies $/ \mu \mathrm{L}$ to $10^{5}$ copies $/ \mu \mathrm{L}$. At a concentration of 100 copies $/ \mu \mathrm{L}$, the OD values were close to the cutoff value for type 31 and type 52 and were $0.5-1.0$ for type 56 , but at a concentration of 500 copies $/ \mu \mathrm{L}$, they showed positive results. Only six types of plasmid (types $16,18,35,45,58$, and 59) were positive at a concentration of 10 copies $/ \mu \mathrm{L}$ (Fig. 2). At the concentration of $10^{4}-10^{5}$ copies $/ \mu \mathrm{L}$, no positive results were obtained by the MPHC method for any of the non-HRHPV or other common genitaltract pathogens, indicating a lack of cross-reactivity between the high-risk and low-risk or genital-tract pathogens. It is believed that the specificity of the screen is determined only by the specificity of the probes. HPV18-transformed cells were used to compare the differences in the MPHC assay results when cells expressing virus or plasmids were analyzed. Although the sensitivity in detecting type 18 HRHPV was the same for the two samples, the HPV18-transformed cell based method was not suitable for evaluation of the differences in the primer and probe sequences between different HPV types.

To determine the epidemic levels of the 13 HRHPV types in high-risk populations in populous cities of China, 1238 cervical specimens were collected as described by the manufacturer of the HCII High-Risk HPV DNA test kit (Digene) [19] and detected by the MPHC and HCII method, respectively. The HCII test was performed with the automated HCII system as described previously [19]. Of the 1238 samples tested, 306 (25\%) were HRHPV positive and $862(70 \%)$ were HRHPV negative when measured by both methods. The two methods had a high concordance rate of $94.3 \%$. A kappa analysis showed a score of 0.859 (SPSS, ver. 18.0, IBM), indicating an "almost perfect" match between these two assays. However, the results for $70(6 \%)$ samples were discordant 
Table 1 Primers and probes used for the MPHC assay of HRHPV

\begin{tabular}{|c|c|c|c|}
\hline Primer/probe & Name & Sequence $\left(5^{\prime}-3^{\prime}\right)$ & $\begin{array}{l}\text { Accession number and nucleotide } \\
\text { position }\end{array}$ \\
\hline \multirow[t]{8}{*}{ Forward primer } & YR8-A & Bio-GCACAGGGTCATAATAATGGTATTTGTTGG & gblFJ202006.1I: bp4-33 \\
\hline & YR8-B & Bio- GCACAGGGACATAATAATGGCATTTGCTGG & gb|U12488.1l: bp1-30 \\
\hline & YR8-C & Bio-GCACAGGGCCACAATAATGGTATTTGTTGG & dbj|AB889493.1I: bp6587-6616 \\
\hline & YR8-D & Bio-GCACAGGGTCATAACAATGGTATTTGCTGG & gb|KF225496.1l: bp149-178 \\
\hline & YR8-E & Bio-GCTCAGGGTTTAAACAATGGTATATGTTGG & gblKC470266.1l: bp6551-6182 \\
\hline & YR8-F & Bio-GCCCAGGGCCACAACAATGGTATATGTTGG & gblKC470239.1l: bp6612-6641 \\
\hline & YR8-G & Bio-GCCCAGGGACATAATAATGGCATTTGTT & emb|AJ620205.1I: bp6696-7 = 6723 \\
\hline & YR8-H & Bio- GCACAGGGTCATAACAATGGTATCTGCTGG & gblKC470221.1l: bp6532-6561 \\
\hline \multirow[t]{9}{*}{ Reverse primer } & YR10-A & Bio-TGAAAAATAAACTGTAAATCATATTCCTC & dbj|AB889494.1I: bp6767-6739 \\
\hline & YR10-B & Bio-TGAAAAATAAACTGTAAATCATATTCTTC & gb|KC991279.1l: bp146-118 \\
\hline & YR10-C & Bio-TGAAAAATAAACTGTAAATCATACTCTTC & gb|EF202168.1l: bp6626-6598 \\
\hline & YR10-D & Bio-TGAAAAATAAACTGTAAATCAAACTCCTC & gb|KC815977.1l: bp182-154 \\
\hline & YR10-E & Bio-TGAAAAATAAACTGTAAATCAAATTCCTC & gblGQ396222.1l: bp72-44 \\
\hline & YR10-F & Bio-TGAAAAATAAACTGTAAATCATACTCCTC & gb|EU056622.1l: bp48-20 \\
\hline & YR10-G & Bio-TGAAAAATAAATTGTAAATCATACTCTTC & emb|HE805662.1I: bp124-96 \\
\hline & YR10-H & Bio-TGAAAAATAAATTGCAAATCATATTCTTC & gb|KC792556.1l: bp1133-1105 \\
\hline & YR10-I & Bio- TGAAAAATAAACTGTATGTCATATTCTTC & gb|JQ041819.1l: bp158-130 \\
\hline \multirow{2}{*}{$\begin{array}{l}\text { Probes for } \\
\text { HPV16 }\end{array}$} & Probe- $16 \mathrm{~F}$ & amine - TTTTTTTTTTTTTTTTAAGGAGTACCTACGACATGG & dbjlAB889494.1I: bp6718-6737 \\
\hline & Probe-16R & amine - TTTTTTTTTTTTTTTGATATGGCAGCACATAATGAC & dbj|AB889494.1I: bp6683-6663 \\
\hline \multirow{2}{*}{$\begin{array}{l}\text { Probes for } \\
\text { HPV18 }\end{array}$} & Probe- $18 \mathrm{~F}$ & amine - TTTTTTTTTTTTTTTTGCTTCTACACAGTCTCCTGTA & gb|KF225496.1l: bp239-259 \\
\hline & Probe-18R & amine - TTTTTTTTTTTTTTTCTTAAATTTGGTAGC ATCATATTG & gb|KF225496.1l: bp289-266 \\
\hline \multirow{2}{*}{$\begin{array}{l}\text { Probes for } \\
\text { HPV31 }\end{array}$} & Probe- $31 \mathrm{~F}$ & amine - TTTTTTTTTTTTTTTCCA CTCCATTTA AАCCATCTG & gb|KC991270.1l:bp36-54 \\
\hline & Probe-31R & amine - TTTTTTTTTTTTTTTCGCCATGTCTTATAAATTGTT & gb|KC991270.1l: bp89-70 \\
\hline \multirow{2}{*}{$\begin{array}{l}\text { Probes for } \\
\text { HPV33 }\end{array}$} & Probe- $33 \mathrm{~F}$ & amine - TTTTTTTTTTTTTTTAT ATATAAGACATGTTGAAGAA & gb|KC706450.1l: bp265-244 \\
\hline & Probe-33R & amine - TTTTTTTTTTTTTTTCTGTCACTAGTTACTTGTGTGCAT & gb|KC706450.1l: bp293-361 \\
\hline \multirow{2}{*}{$\begin{array}{l}\text { Probes for } \\
\text { HPV35 }\end{array}$} & Probe- $35 \mathrm{~F}$ & amine - TTTTTTTTTTTTTTTCTGCTGTGTCTTCTAGTGACAG & gb|KC991278.1l: bp53-74 \\
\hline & Probe-35R & amine - TTTTTTTTTTTTTTTCACAGACATATTTGTACTACGGG & gb|KC991278.1l: bp48-26 \\
\hline \multirow{2}{*}{$\begin{array}{l}\text { Probes for } \\
\text { HPV39 }\end{array}$} & Probe-39F & amine - TTTTTTTTTTTTTTTTAGAGTCTTCCATACCTTCTAC & gb|KC470249.1l:bp6683-6703 \\
\hline & Probe-39R & amine - TTTTTTTTTTTTTTTGCCTGGTATATTCCTTAAACTTA & gb|KC470249.1l:bp6738-6716 \\
\hline \multirow{2}{*}{$\begin{array}{l}\text { Probes for } \\
\text { HPV45 }\end{array}$} & Probe $45 \mathrm{~F}$ & amine - TTTTTTTTTTTTTTTTGACCCTACTAAGTTTAAGCAG & gb|KC470256.1l: bp6676-6697 \\
\hline & Probe-45R & amine - TTTTTTTTTTTTTTTGCACAGGATTTTGTGTAGAG & gb|KC470256.1l: bp6665-6646 \\
\hline \multirow{2}{*}{$\begin{array}{l}\text { Probes for } \\
\text { HPV51 }\end{array}$} & Probe- $51 \mathrm{~F}$ & amine - TTTTTTTTTTTTTTTTATTAGCACTGCCACTGCTG & gblS40272.1l: bp16-36 \\
\hline & Probe-51R & amine - TTTTTTTTTTTTTTTCTTGGAGTAAATGTTGGGG & gblS40272.1l: bp77-54 \\
\hline \multirow{2}{*}{$\begin{array}{l}\text { Probes for } \\
\text { HPV52 }\end{array}$} & Probe- $52 \mathrm{~F}$ & amine - TTTTTTTTTTTTTTTGGAATACCTTCGTCATGG & gb|KF225497.11: bp987-1009 \\
\hline & Probe-52R & amine - TTTTTTTTTTTTTTTCCTTTTTAACCTCAGCAC & gb|KF225497.1I: bp1040-1018 \\
\hline \multirow{2}{*}{$\begin{array}{l}\text { Probes for } \\
\text { HPV56 }\end{array}$} & Probe- $56 \mathrm{~F}$ & amine - TTTTTTTTTTTTTTTTGACTATTAGTACTGCTACAGAA & gb|KC815983.1l: bp75-96 \\
\hline & Probe-56R & amine - TTTTTTTTTTTTTTTCG TGCATCATATTTACTTAACTG & gb|KC815983.1l: bp119-97 \\
\hline \multirow{2}{*}{$\begin{array}{l}\text { Probes for } \\
\text { HPV58 }\end{array}$} & Probe- $58 \mathrm{~F}$ & amine - TTTTTTTTTTTTTTGACATTATGCACTGAAGTAACTAAG & dbj|AB819279.1I: bp6668-6692 \\
\hline & Probe-58R & amine - TTTTTTTTTTTTTTTCATATTCСТTAАAАTTATCATT & dbj|AB819279.1I: bp6729-6708 \\
\hline \multirow{2}{*}{$\begin{array}{l}\text { Probes for } \\
\text { HPV59 }\end{array}$} & Probe- $59 \mathrm{~F}$ & amine - TTTTTTTTTTTTTTTCCTAATGTATACACACCTACCAG & gb|KC470266.1l: bp6662-6684 \\
\hline & Probe-59R & amine - TTTTTTTTTTTTTTTAGAAGAAGTAGTAGAAGCACA & gb|KC470266.1l: bp6658-6638 \\
\hline \multirow{2}{*}{$\begin{array}{l}\text { Probes for } \\
\text { HPV68 }\end{array}$} & Probe- $68 \mathrm{~F}$ & amine - TTTTTTTTTTTTTTTCTACTACTGAATCAGCTGTACC & gb|KC470283.1l: bp6544-6565 \\
\hline & Probe-68R & amine - TTTTTTTTTTTTTTTCCTTAAATTTATTAGGATCATA & gb|KC470283.1l:bp6594-6573 \\
\hline
\end{tabular}




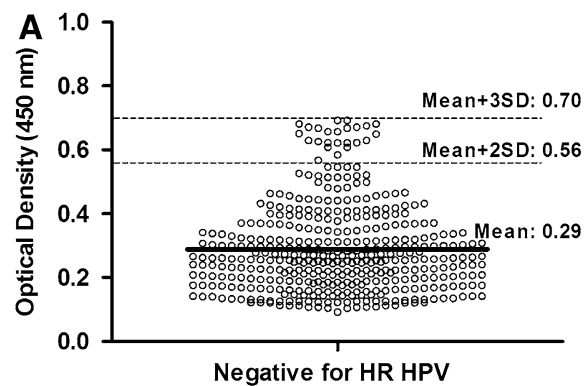

Fig. 1 Determining the negative and positive cutoff values. A) Estimation of the cutoff value with 400 samples negative for HRHPV. The negative and positive cutoff values were estimated by calculating the mean negative $\mathrm{OD}_{450}$ values plus two or three standard deviations to be 0.56 and 0.70 , respectively. B) Validation of the cutoff levels by MPHC assays performed on samples classified as negative or positive

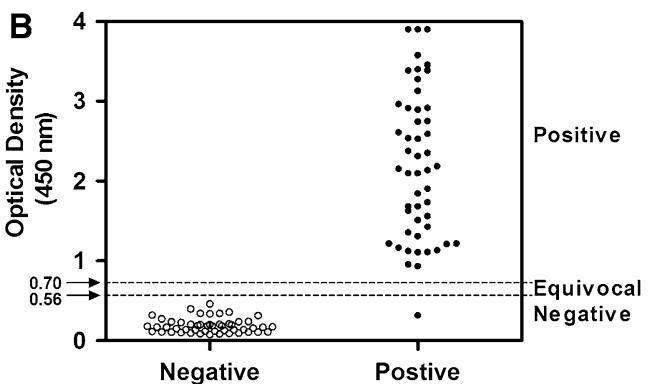

by the HCII assay. Using the cutoff value derived from the analysis described above as the criterion for positive specimens, more than $95 \%$ of samples were identified as positive. The positive samples in the negative region were verified by DNA sequencing analysis and resolved as positive. This scatter plot was constructed with GraphPad Prism 5.0 (GraphPad Software, San Diego, CA, USA)
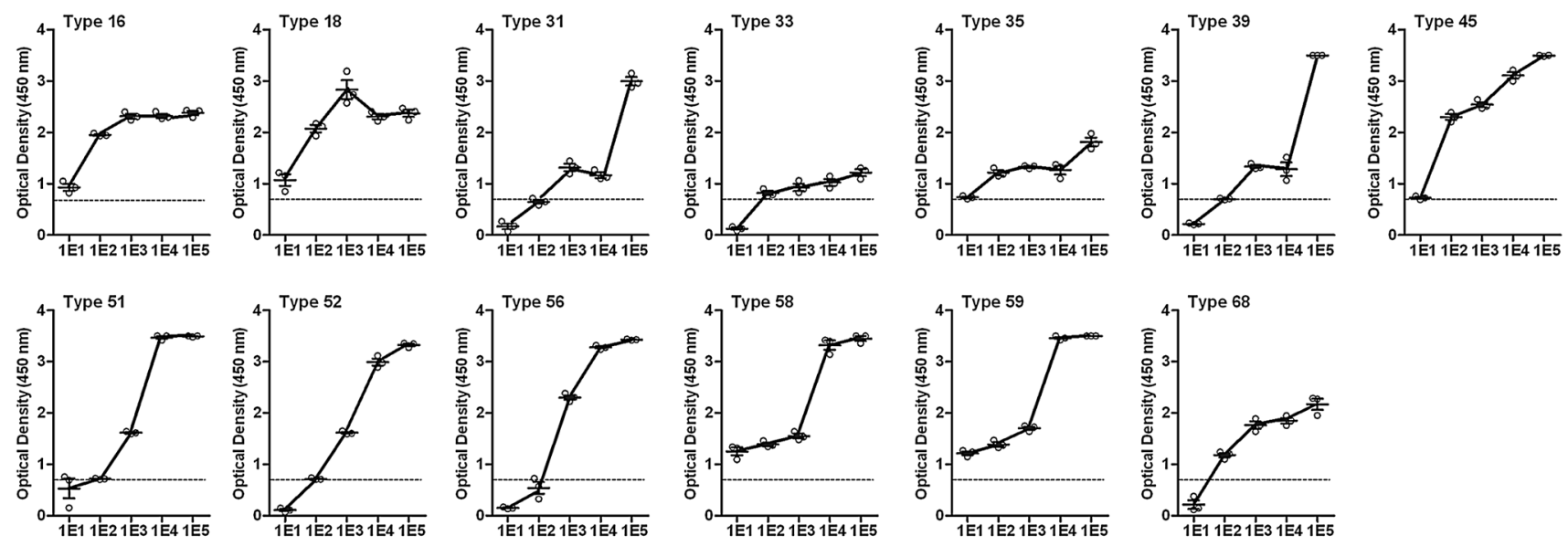

Plasmid concentration (copies/ $\mu \mathrm{L}$ )

Fig. 2 Detection limit of the MPHC assay for each of the 13 HRHPV plasmids at concentrations ranging from 10 copies $/ \mu \mathrm{L}$ to $10^{5}$ copies/ $\mu \mathrm{L}$. The symbol $(\mathrm{O})$ shows the $\mathrm{OD}_{450}$ value. The dotted line

between the two assays. To confirm the type of virus present in the specimens with discordant results in HCII and MPHC, the samples were subjected to DNA sequencing, which showed that MPHC was correct for 33 samples, and HCII was correct for 6 samples. Twentyseven specimens produced no HPV sequence results, and these were assumed to be HPV negative.

Based on these results, the sensitivity and specificity of the MPHC assay were 98.5\% (339/344) and 97.3\% (870/ 894), respectively. The HRHPV prevalence rate, when measured by MPHC, was $29.3 \%$ (363/1238) in cervical specimens sampled from hospitals in three different regions of China. Although DNA sequencing is regarded as the gold-standard technique, there were still false negatives for the 24 samples diagnosed as positive by MPHC but negative by HCII assay (HCII-MPHC+), which were represents the positive cutoff value, estimated to be 0.7. Each data point represents the average of three duplicated experiments

deemed to be negative based on sequencing failure, even if their MPHC detection values were more than 1.5. Because most sequencing failures are caused by the condition of the sample, including too little DNA, the $29.3 \%$ positive detection rate with the MPHC method, which was higher than that with the HCII method, may be closer to the true positive rate. Therefore, the prevalence of HRHPV infection in female outpatients was between $27.8 \%$ and $29.3 \%$ in the regions of China examined, which was higher than those in the report by Li et al. [7].

The most prevalent HPV screening method is the HCII assay, which has been approved by the U.S. FDA. This method is validated by the strong agreement between its results and those of cytological or histological assays [20, 21]. However, the high cost of the assay makes it unfeasible for the routine mass screening of cervical infections in 
resource-poor areas of developing countries [22]. The present study suggested that the MPHC method is similar to HCII in terms of its specificity and sensitivity. In this study, using the same collection method, we eliminated the effects of the sample collection method. Therefore, the comparative results reflect the true efficiency of the PCR amplification, hybridization, and signal development achieved with the two methods.

A previous study revealed false positive results with the HCII method in detecting these 13 HRHPV types when 1 RLU/CO (relative light unit) was used as the cutoff value $[23,24]$. The most frequently detected false positives occurred with HRHPV types 53, 66, 67, and 73 [25]. In this study, three of the five HCII-positive but MPHC-negative $\left(\mathrm{HCII}^{+} \mathrm{MPHC}^{-}\right.$) samples were confirmed by sequencing to be true negatives, and they belonged to type 66 , whereas the other two samples belonged to type 73 . In the same analysis, three of the five $\mathrm{HCII}^{+} \mathrm{MPHC}^{-}$samples were confirmed by sequencing to be true positives and belonging to type 52, whereas the other two samples were types 33 and 35. The sequencing results showed that the HPV types of the $\mathrm{HCII}^{-} \mathrm{MPHC}^{+}$samples were limited to HPV types 16 (12/33), 58 (7/33), 59 (7/33), 35 (3/33), 45 (3/33) and 18 (1/33). These results suggested that the MPHC method was more sensitive than HCII in detecting HPV types 16,35 , 45,58 , and 59, and that the detection efficiency for type 52 requires further improvement. The MPHC assay for plasmids was slightly less sensitive in detecting three HPV types (types 31,52 , and 56) than in detecting the other types tested. The OD values at plasmid concentrations of 100 copies/ $\mu \mathrm{L}$ were close to the cutoff value for type 31 and type 52 and were in the range of $0.5-1.0$ for type 56 . Therefore, their unstable concentration was 100 copies $/ \mu \mathrm{L}$. Despite finding no false-negative type 31 or type $56 \mathrm{HPV}$ in the clinical samples (although we did find false-negative type 52), we must increase the sensitivity of their detection in this assay.

In summary, a sensitive, specific, low-cost, highthroughput PCR-based method was developed to detect 13 types of HRHPV in patient specimens. Although this hybridization process takes more time than the real-time PCR assay, it is a sensitive and specific assay that should enhance the study of HRHPV infections and the prevention of cervical cancer among a larger population. However, much effort should be made to standardize the established MPHC before it is widely applied clinically.

Acknowledgments We extend our sincere thanks to Dr. Lu of the Third Xiangya Hospital of Central South University, Dr. Song of the Chinese PLA General Hospital, Director Shi of Boai Hospital, and Dr. Xie of Shenzhen Maternal and Child Health Hospital, who provided valuable cervical specimens. This study was supported by the National High Technology Research and Development Program 863 of China (2011AA02A115).
Conflict of interest The authors declare that they have no conflict of interest.

Open Access This article is distributed under the terms of the Creative Commons Attribution License which permits any use, distribution, and reproduction in any medium, provided the original author(s) and the source are credited.

\section{References}

1. Kim J, Kim BK, Lee CH, Seo SS, Park SY, Roh JW (2012) Human papillomavirus genotypes and cofactors causing cervical intraepithelial neoplasia and cervical cancer in Korean women. Int J Gynecol Cancer 22(9):1570-1576

2. Williams VM, Filippova M, Soto U, Duerksen-Hughes PJ (2011) HPV-DNA integration and carcinogenesis: putative roles for inflammation and oxidative stress. Future Virol 6(1):45-57

3. Lehoux M, D'Abramo CM, Archambault J (2009) Molecular mechanisms of human papillomavirus-induced carcinogenesis. Public Health Genomics 12(5-6):268-280

4. Pim D, Banks L (2010) Interaction of viral oncoproteins with cellular target molecules: infection with high-risk vs low-risk human papillomaviruses. Apmis 118(6-7):471-493

5. Rogovskaya SI, Shabalova IP, Mikheeva IV, Minkina GN, Podzolkova NM, Shipulina OY, Sultanov SN, Kosenko IA, Brotons M, Buttmann N, Dartell M, Arbyn M, Syrjanen S, Poljak M (2013) Human papillomavirus prevalence and type-distribution, cervical cancer screening practices and current status of vaccination implementation in Russian Federation, the Western Countries of the former Soviet Union Caucasus Region and Central Asia. Vaccine 31(Suppl 7):H46-H58

6. Forman D, de Martel C, Lacey CJ, Soerjomataram I, LortetTieulent J, Bruni L, Vignat J, Ferlay J, Bray F, Plummer M, Franceschi S (2012) Global burden of human papillomavirus and related diseases. Vaccine 30(Suppl 5):F12-F23

7. Li J, Huang R, Schmidt JE, Qiao YL (2013) Epidemiological features of human papillomavirus (HPV) infection among women living in Mainland China. Asian Pac J Cancer Prev 14(7):4015-4023

8. Shan J (2014) Cervical cancer increases among young Chinese women. http://news.xinhuanet.com/english/china/2014-04/25/c_ 133289589.htm

9. Jeong NH, Lee NW, Kim HJ, Kim T, Lee KW (2009) High-risk human papillomavirus testing for monitoring patients treated for high-grade cervical intraepithelial neoplasia. J Obstet Gynaecol Res 35(4):706-711

10. Ronco G, Giorgi-Rossi P, Carozzi F, Confortini M, Dalla Palma P, Del Mistro A, Ghiringhello B, Girlando S, Gillio-Tos A, De Marco L, Naldoni C, Pierotti P, Rizzolo R, Schincaglia P, Zorzi M, Zappa M, Segnan N, Cuzick J (2010) Efficacy of human papillomavirus testing for the detection of invasive cervical cancers and cervical intraepithelial neoplasia: a randomised controlled trial. Lancet Oncol 11(3):249-257

11. zur Hausen H (2009) Papillomaviruses in the causation of human cancers-a brief historical account. Virology 384:260-265

12. Persano S, Valentini P, Kim JH, Pompa PP (2013) Colorimetric detection of human papilloma virus by double isothermal amplification. Chem Commun (Camb) 49(90):10605-10607

13. Nilyanimit P, Wanlapakorn N, Niruthisard S, Pohthipornthawat N, Karalak A, Laowahutanont P, Phanuphak N, Gemma N, Poovorawan Y (2013) Detection of human papillomavirus in male and female urine by electrochemical DNA chip and PCR sequencing. Asian Pac J Cancer Prev 14(9):5519-5525 
14. Eder PS, Lou J, Huff J, Macioszek J (2009) The next-generation Hybrid Capture High-Risk HPV DNA assay on a fully automated platform. J Clin Virol 45(Suppl 1):S85-S92

15. Ju Z, Yane G, Ding L, Guowu Y, Yanhai G, Yonglan L (2009) Asymmetric GP5 +/6 + PCR and hybridization with fluorescence polarization assay of 15 human papillomavirus genotypes in clinical samples. J Clin Virol 44(2):106-110

16. Salimovic-Besic I (2007) Evaluation of hybrid capture 2 HPV DNA test and two variants of polymerase chain reaction (PCRPGMY11/ PGMY09 and PCR-CPI/CPIIG) according to HPV types. Med Arh 61(3):135-137

17. Chaiwongkot A, Pientong C, Ekalaksananan T, Kongyingyoes B, Thinkhamrop J, Yuenyao P, Sriamporn S (2007) Evaluation of primers and PCR performance on HPV DNA screening in normal and low grade abnormal cervical cells. Asian Pac J Cancer Prev 8:279-282

18. Adefuye PO, Broutet NJ, de Sanjose S, Denny LA (2013) Trials and projects on cervical cancer and human papillomavirus prevention in sub-saharan Africa. Vaccine 31(Suppl 5):F53-F59

19. Szarewski A, Mesher D, Cadman L, Austin J, Ashdown-Barr L, Ho L, Terry G, Liddle S, Young M, Stoler M, McCarthy J, Wright C, Bergeron C, Soutter WP, Lyons D, Cuzick J (2012) Comparison of seven tests for high-grade cervical intraepithelial neoplasia in women with abnormal smears: the Predictors 2 study. J Clin Microbiol 50(6):1867-1873

20. Kuebler DLIA, Blenc AM, Wilbur DC (2007) A peer comparison program for the quality assurance of human papillomavirus DNA detection using the Digene Hybrid Capture II/SurePath method shows excellent analytic interlaboratory correlation. Cancer 111(5):339-343

21. Tan JH, Garland SM, Tabrizi SN, Moore EE, Danielewski JA, Quinn MA (2013) Hybrid Capture II testing for high-risk human papillomavirus DNA in the follow-up of women treated for highgrade cervical intraepithelial neoplasia. J Low Genit Tract Dis 17(3):308-314

22. Carestiato FN, Silva KC, Dimetz T, Oliveira LH, Cavalcanti SM (2006) Prevalence of human papillomavirus infection in the genital tract determined by hybrid capture assay. Braz J Infect Dis 10(5):331-336

23. Rebolj M, Bonde J, Njor SH, Lynge E (2011) Human papillomavirus testing in primary cervical screening and the cut-off level for hybrid capture 2 tests: systematic review. Bmj 342:d2757d2766

24. Guyot A, Karim S, Kyi MS, Fox J (2003) Evaluation of adjunctive HPV testing by Hybrid Capture II in women with minor cytological abnormalities for the diagnosis of CIN $2 / 3$ and cost comparison with colposcopy. BMC Infect Dis 3:23-30

25. Peyton CL, Schiffman M, Lorincz AT, Hunt WC, Mielzynska I, Bratti C, Eaton S, Hildesheim A, Morera LA, Rodriguez AC, Herrero R, Sherman ME, Wheeler CM (1998) Comparison of PCR- and hybrid capture-based human papillomavirus detection systems using multiple cervical specimen collection strategies. J Clin Microbiol 36(11):3248-3254 